\title{
CRISPR-induced double-strand breaks trigger recombination between homologous chromosome arms
}

\author{
Erich Brunner ${ }^{1, \star}\left(\mathbb{D}\right.$, Ryohei Yagi $^{2, \star}\left(\mathbb{D}\right.$, Marc Debrunner $^{1} \mathbb{D}$, Dezirae Beck-Schneider ${ }^{1}$, Alexa Burger ${ }^{1}$, Eliane Escher ${ }^{1}$, \\ Christian Mosimann', George Hausmann ${ }^{1}$, Konrad Basler ${ }^{1}$
}

\begin{abstract}
CRISPR-Cas9-based genome editing has transformed the life sciences, enabling virtually unlimited genetic manipulation of genomes: The RNA-guided Cas9 endonuclease cuts DNA at a specific target sequence and the resulting double-strand breaks are mended by one of the intrinsic cellular repair pathways. Imprecise double-strand repair will introduce random mutations such as indels or point mutations, whereas precise editing will restore or specifically edit the locus as mandated by an endogenous or exogenously provided template. Recent studies indicate that CRISPR-induced DNA cuts may also result in the exchange of genetic information between homologous chromosome arms. However, conclusive data of such recombination events in higher eukaryotes are lacking. Here, we show that in Drosophila, the detected Cas9-mediated editing events frequently resulted in germline-transmitted exchange of chromosome arms-often without indels. These findings demonstrate the feasibility of using the system for generating recombinants and also highlight an unforeseen risk of using CRISPR-Cas9 for therapeutic intervention.
\end{abstract}

DOI 10.26508/Isa.201800267 | Received 6 December 2018 | Revised 29 May 2019 | Accepted 29 May 2019 | Published online 13 June 2019

\section{Introduction}

CRISPR-Cas9-based genome editing has revolutionized genetic research, triggering the development of a plethora of technologies and applications that provide unprecedented control over genes in a growing list of model species (1, 2, 3, 4, 5, 6, 7, 8). CRISPR systems allow us to edit, engineer, or regulate genomes, hold great promise for clinical applications, and are likely to be used to treat diseases with genetic underpinnings, including cancer $(9,10)$. Genome editing is achieved by precisely targeting the nuclease activity of a modified bacterial protein (Cas9) via a user-defined guide RNA to a specific DNA sequence (1). The resulting DNA double-strand breaks (DSBS) are repaired either by the error-prone nonhomologous end joining (NHEJ) or homology-directed repair (11). For refined and precise genome editing purposes, homology-directed repair is harnessed to copy a specific DNA template (single-stranded or double-stranded) into the target site $(2,9,12,13)$. In contrast, NHEJ ligates the two broken ends of the DNA without a donor template, often resulting in random insertions or deletions (indels) that can disrupt coding sequences at the target site (for review see reference 14). However, with directly ligateable ends, NHEJ may lead to accurate repair of close and concurrent DSBS $(15,16,17,18)$, also when induced by Cas $9(19,20,21$, 22). The ability of CRISPR-Cas9 to introduce several concurrent DSBS at defined positions has enabled engineering of tumor-associated chromosomal translocations resembling those observed in cancers, and hence to establish and test novel in vitro and in vivo tumor models $(2,23,24,25)$. Sadhu et al. (26) leveraged the CRISPR-Cas9 system to produce other chromosomal rearrangements, generating targeted mitotic recombination events in yeast to enable the fine mapping of trait variants. The authors deliberately induced a single DSB in one of the homologous chromosomes in a diploid yeast strain and achieved homologous recombination-based "loss of heterozygosity" events within $20 \mathrm{~kb}$ of the target site. Additional reports suggest that recombination in mitotic cells is not restricted to yeast but may also occur in other species such as houseflies (27) and tomatoes (28). What is currently missing is a solid confirmation of such events and data on their frequency in different species.

Here, we set out to examine the occurrence and frequency of genetic exchanges between homologous chromosome arms initiated by Cas9-induced DSBs. We show that Cas9-triggered DSBS induce germline-transmitted recombination between homologous chromosome arms in up to $39 \%$ of the CRISPR events in Drosophila. Although these findings expand the tool-box of CRISPR-based genome manipulation in research, they also raise concerns about the use of gene editing in therapeutic settings.

\section{Results}

\section{CRISPR/Cas9 cuts induce recombination events}

NHEJ is a major repair mechanism triggered by CRISPR-Cas9induced DSBs in Drosophila $(29,30)$. Leveraging on this, we developed

${ }^{1}$ Institute of Molecular Life Sciences, University of Zurich, Zurich, Switzerland ${ }^{2}$ Institute of Molecular Systems Biology, Eidgenössische Technische Hochschule Zurich, Zurich, Switzerland 
a system for activating transgene expression through NHEJ-based repair. The system, which we named CIGAR (CRISPR-Induced Gene Activator), allows activation of transgene expression after CRISPRinduced DSBs. The principle of the CIGAR system is based on activation of gene expression if, and only if, a unique CRISPR-Cas9 target sequence has been cleaved and rearranged by NHEJ (Fig 1). CIGAR consists of four elements: (i) the ubiquitin-p63E promoter to drive gene expression in every cell (31), (ii) a so-called "shifter" sequence, (iii) a flexible linker sequence inserted 3 ' of the shifter sequence (32), and (iv) a reporter CDNA (lacking a translational start codon) followed by the 3'UTR of the Drosophila tubulin $\alpha 1$ gene. The functional core of the CIGAR reporter lies within the shifter sequence, which contains optimized translational START codons covering all three frames upstream of a unique 20-nt CRISPR target sequence (33, 34). Each initiation codon is blocked downstream by a corresponding in-frame STOP codon. Importantly, the most 5' STOP codon, named STOP ${ }^{T}$ ( $T$ for target), is in-frame with the downstream ORF and resides within the unique 20-nt gRNA target, starting 4 nt upstream of the PAM sequence. Activation of CIGAR is achieved by Cas9-induced DNA cleavage within the STOP ${ }^{T}$ codon. The induced DSBs will then be mended by NHEJ-mediated repair concomitantly severing or eliminating the STOPT codon. The resulting indel leads to repositioning of the upstream ATGs relative to the ORF, that is, causing one of the ATGS to be "shifted in-frame" with the ORF.

Two variants of this CIGAR tool box, that is, CIGAR ${ }^{\text {eGFP }}$ or CIGAR ${ }^{\text {mCherry }}$ are depicted in Figs 1 and 2A. Further details of the CIGAR system will be submitted elsewhere. When we molecularly analyzed the shifter sequences in the progeny of females carrying the two
CIGAR transgenes in trans (genotype: nos-Cas9, CIGAR ${ }^{\text {eGFP } / C I G A R^{\text {mCherry; }}}$; U6:3-sgRNA ${ }^{\operatorname{CIGAR}(1,2) /+}$ ), we found that in some of the F1 animals, the CRISPR target sequence of the CIGAR ${ }^{\text {mCherry }}$ reporter became located $5^{\prime}$ of the eGFP ORF and vice versa. Sequence analysis of 84 animals from different crosses revealed a total of 26 animals in which the sequences on one side of the DSB had been exchanged (Figs 2B, S1, and S2 and Table S1). The site of the DSB was in part marked by indels. From these results, we concluded that in these animals, recombination events had occurred at the site of the Cas9-induced DSBs. We would like to emphasize that the term recombination is used here to describe the exchange of genetic material between homologous chromosome arms initiated by CRISPR-induced DSBS. Because Cas 9 activity may often cause the break of both homologs, such recombination events may not only be based on homologous recombination but may also result by breakage/fusion events involving NHEJ that lead to a crosswise ligation of the chromosome $\operatorname{arms}(15,16,17,18,19,20,21,22)$. From our results, however, we cannot infer which repair mechanism was involved in the exchange of genetic information between homologous chromosome arms.

In the experiment described above, the CIGAR transgenes were located at position 5D on the X-chromosome that shows native recombination activity in the female germline (35). To further test if CRISPR-Cas9 could induce efficient site-specific recombination, we turned to the fourth chromosome for which normally no naturally occurring recombination is observed $(36,37,38)$. We first generated transgenic animals harboring the CIGAR ${ }^{\text {eGFP }}$ and CIGAR ${ }^{\text {mCherry }}$ construct at position $102 \mathrm{~F}$ on the two homologous arms of chromosome 4 , in addition to nos-Cas9 and U6:3-sgRNA ${ }^{C I G A R(1,2)}$. Such
A

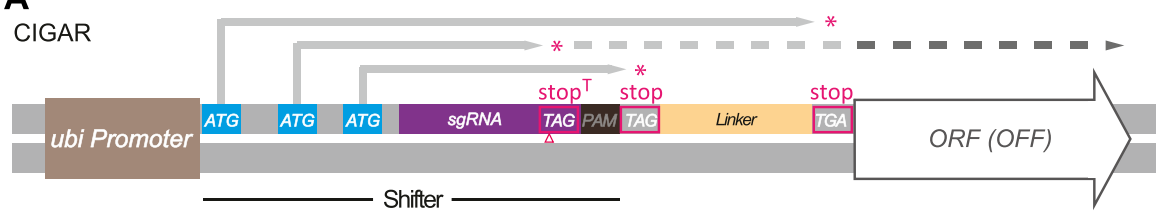

B
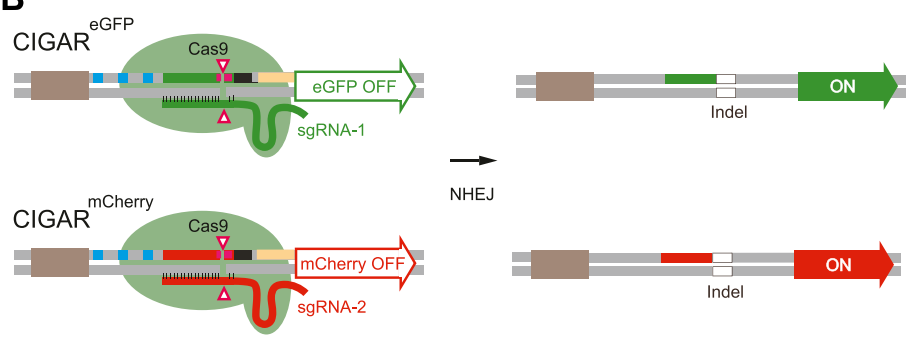

$\longrightarrow$
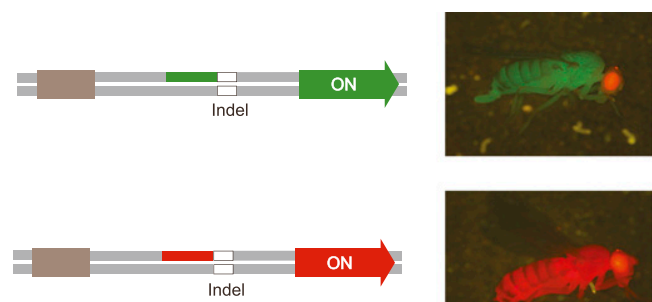

\section{C}

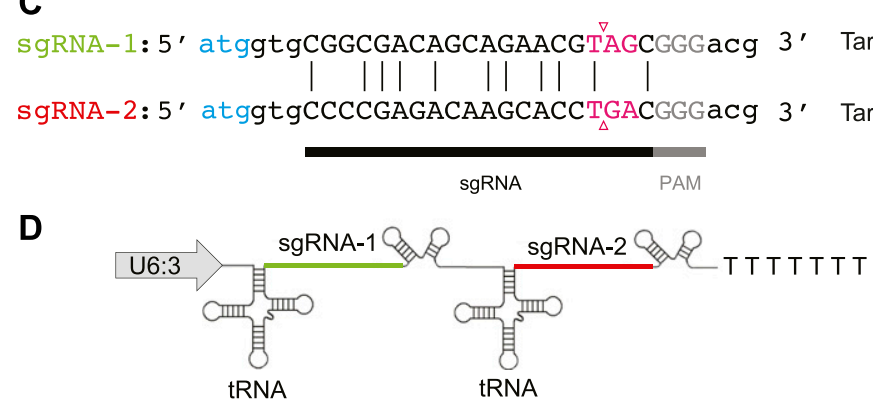

Figure 1. CIGAR design and activation.

(A) CIGAR consists of four elements: 1. Ubiquitin-p63E promoter (brown box), 2. "shifter" sequence, 3. linker sequence (orange), 4. reporter CDNA (ORF) lacking a translational start site followed by the tubulin 3'UTR (grey open arrow). The shifter sequence contains optimized translation initiation codons covering all three frames (light blue boxes) and a guide RNA target region (purple box) followed by a protospacer adjacent motif (PAM) (black box). In the inactive CIGAR,

translation from each ATG (grey arrows) is terminated 5' of the ORF by a STOP codon (red asterisks) preventing the translation of the downstream ORF. (B) Activation of two CIGAR variants harboring either an eGFP or a monomeric Cherry (mCherry). Activation is achieved by Cas9-induced DNA cleavage within stop ${ }^{\top}$ (pink), the most upstream STOP codon which is in-frame with the downstream ORF. The resulting double-strand break (DSB; the putative site of the DSB, 3 bp upstream of the PAM site is indicated with a pink, open arrowhead) is mended by NHEJ-mediated repair concomitantly eliminating stop ${ }^{\top}$ (indel; open white box) and shifting one of the ATGs in-frame with the ORF. Flies that inherited a translationally activated CIGAR appear uniformly green or red. The eye-specific red fluorescence marks the attP target site into which the CIGAR constructs have been inserted. (C) Comparison of the 20-nt sgRNA target sequences of CIGAR ${ }^{\text {EGFP }}$ and CIGAR $^{\text {mCherry }}$, respectively. The 9-bp substitutions in the two target sites are indicated. (D) For specific and simultaneous targeting of both reporters, a tRNAspaced tandem array (U6:3-sgRNA $\operatorname{CIGAR}(1,2)^{2}$ harboring sgRNA-1 (targeting CIGAR ${ }^{\text {EGFP) }}$ ) and sgRNA-2 (targeting CIGAR $\left.{ }^{\text {mCherry }}\right)$ is used. 
A

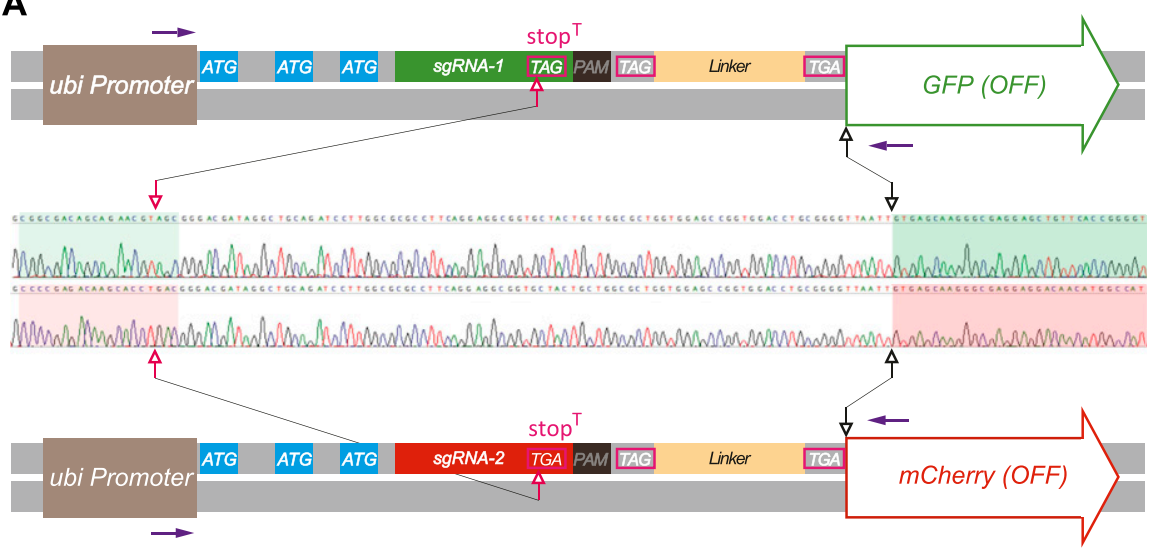

B

SgRNA-2 $\nabla$ PAM

Linker

eGFP

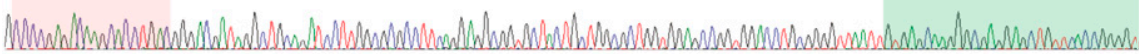

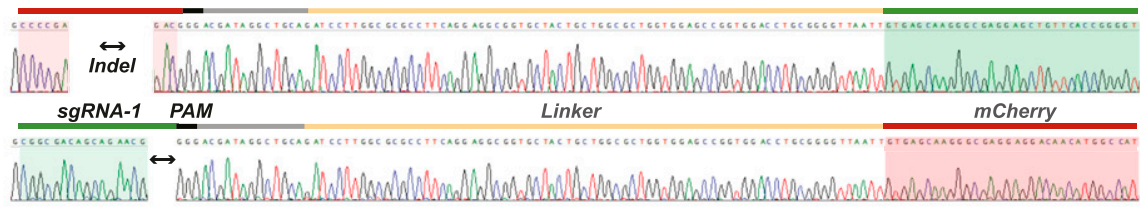

Figure 2. Detailed CIGAR ${ }^{\text {eGFP }}$ and CIGAR ${ }^{\text {mCherry }}$ reporter design and illustration of recombination events on the sequence level.

(A) Design and sequence details of un-CRISPRed CIGAR $^{\text {eGFP }}$ (top) and CIGAR ${ }^{\text {mCherry }}$ reporters (bottom). The sequences of the sgRNAs and the ORFs are shaded in green and red, respectively. Note that except for the sgRNAs and the ORFs, the sequences of the reporters are identical. The targeted STOP codon (stop ${ }^{\top}$; pink) differ in sequence. The CRISPR target sites are delineated in the sequence context (pink, open arrows). Analysis of the shifter sequence is performed using primer pairs specific for the Ubi promoter and the $5^{\prime}$ end of the respective ORF (purple arrows). (B) The shifter region of flies harboring a single copy of one of the CIGAR reporters on the $X$ chromosome (attP 5D) was analyzed by single fly PCR and Sanger sequencing. Shown are recombination events from a CIGAR ${ }^{\text {eGFP }} /$ CIGAR ${ }^{\text {mCherry }}$ co-targeting experiment visualized on the sequence level. As in (A), the sequences of the sgRNAs and the ORFs are shaded in green and red, respectively. Recombinants exhibit a rearranged sequential arrangement (green-red or red-green) of sgRNA and reporter cDNA. Note that recombination events may or may not be accompanied by indels at the target site. (c) Co-targeting experiments using CIGAR reporters on the fourth chromosome (attP 102F). 172 animals were analyzed by single fly PCR and Sanger sequencing. The yellow sections represent the number of recombinants with or without indel.

\section{C}

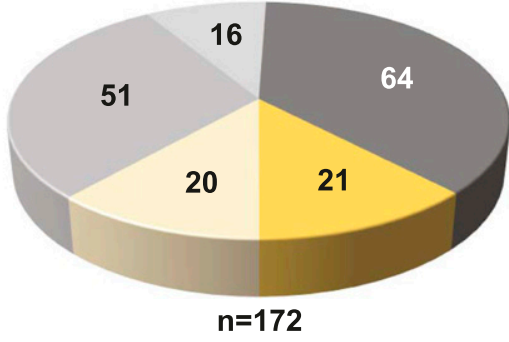

UnCRISPRed

Unreadable sequences

Indels (no recombination)

Recombinations without indels

Recombinations with indels individuals were crossed to $y w$ animals, and their offspring were scored for recombination events (Fig 2C, see the Materials and Methods section for details). Animals were randomly picked from 13 crosses immediately after hatching and analyzed for the sequences flanking the target site (Tables S2 and S3). This revealed that in 41 of 156 animals, recombination events had occurred (Fig 2B; for details see the Materials and Methods section). Thus, as described above for the X chromosome, numerous CRISPR-mediated recombination events were observed on the fourth chromosome (39\% of the detected Cas9-triggered events, i.e., 41 of 105 CRISPR events).

\section{CRISPR-induced recombination between two distant phenotypic markers}

In the above experiments, recombination was induced between homologous chromosome arms for which the nt at the Cas9 target site as well as the flanking sequences (coding for the fluorescent proteins) differed. However, the recombination events could only be demonstrated by sequence analysis of the immediate vicinity of the CRISPR site; more distant phenotypic markers were not present on these chromosomes. Therefore, we could not rule out that, at least in some cases, other mechanisms, such as gene conversion, were responsible for the observed sequence exchange between the two CIGAR reporters in trans (39).

To confirm that indeed a complete exchange of the homologous chromosome arms occurs distal to the CRISPR-Cas9-induced DSBS, we used two visible markers separated by more than $100 \mathrm{~kb}$ (Figs $3 \mathrm{~A}$ and S3): the $\mathrm{w}^{+}$-marked CIGAR ${ }^{\text {mCherry, } 102 F, w^{+}}$and the recessive viable mutation $s v^{\text {spa-pol }}$ close to the tip of the right arm on chromosome 4. We selected the Cas9 target site, targeted with sgRNA-3, in the 3'UTR of the toy gene residing about $18 \mathrm{~kb}$ downstream of the CIGAR ${ }^{m C h e r r y, 102 F, w_{+}}$ transgene insertion site. To induce recombination at the target site (Fig $3 \mathrm{~A}$, TR; see the Materials and Methods section for more experimental details), we injected $y w ; C I G A R^{m C h e r r y, 102 F, w^{+}} / D p(1 ; 4) 1021, y^{+}$, sv $^{\text {spa-pol }}$ embryos with active Cas9-sgRNA RNP complexes containing recombinant Cas9 and in vitro-translated sgRNA-3 (Fig 3B) (40, 41). G0 
A
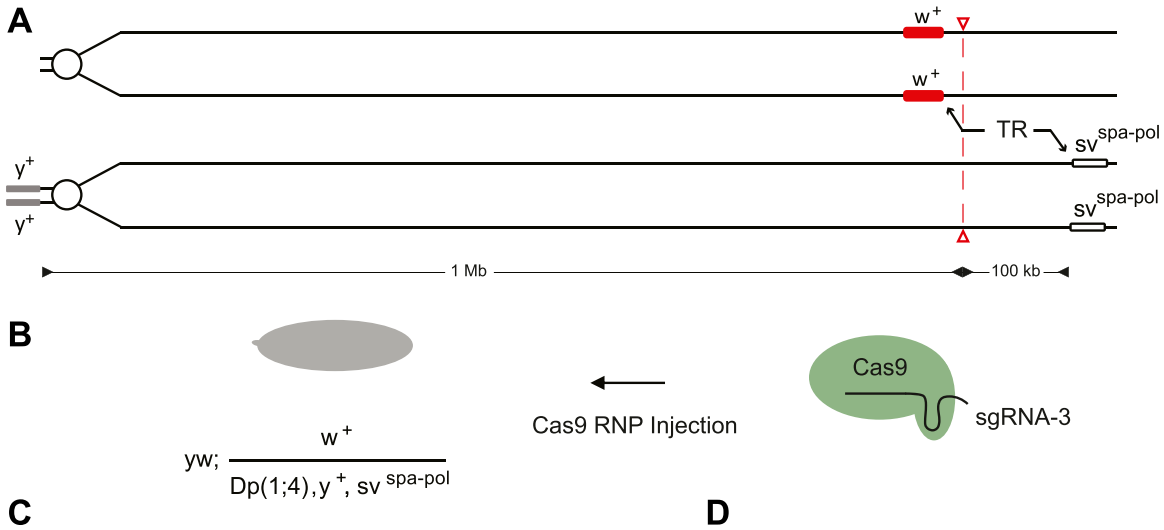

C
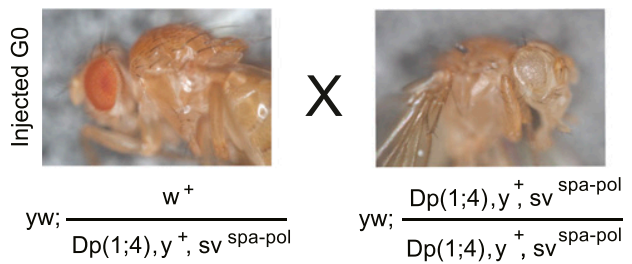

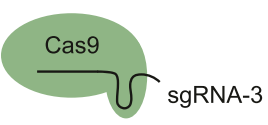

Cas9 RNP Injection

D

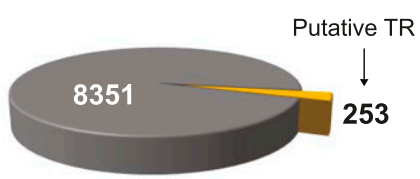

Total F1 animals screened: $\mathrm{n}=8604$
Figure 3. Cas9-induced recombination between two phenotypic markers on the fourth chromosome.

(A) Each of the two markers $\left(w^{+}[\right.$mini-white $]$and $\left.s v^{\text {spa-pol }}\right)$ is located on a different homologous chromosome. The two markers are separated by about $100 \mathrm{~kb}$. The $s v^{\text {spa-pol }}$ chromosome is marked with $\mathrm{y}^{+}$due to a duplication of $\mathrm{X}$ chromosomal material to the short, left arm of chromosome four. The Cas9 cut site is represented by a red dashed line and red open arrow heads. The repair of CRISPR-induced DSB may lead to targeted

recombination events (TR) between the two markers. (B) Embryos with the genotype yw; CIGAR ${ }^{\text {mCherry, } 102 F, w^{+}} /$ $D p(1 ; 4) 1021, y^{+}, s v^{\text {spa-pol }}$ were injected with recombinant Cas9 RNPs containing in vitro-translated sgRNA-3. (C) Cas9 RNP injected G0 animals are backcrossed to animals with the genotype $y w ; D p(1 ; 4) 1021, y^{+}, s v^{\text {spa-pol }}$ $D p(1 ; 4) 1021, y^{+}, s v^{\text {spa-pol }}$ to be able to visually score putative recombinants. The phenotype of the animals is shown. (D) A total of 8,604 animals were screened and 253 putative recombinants were recovered. (E) Unrecombined animals (UR) appeared phenotypically as $\mathrm{y}^{+} ; \mathrm{w}^{+}$(UR-A) or $\mathrm{y}^{+} ; \mathrm{sv}^{\text {spa-pol }}$ (UR-B). Putative recombinants (boxed) presented either as $\mathrm{y}^{+} ; \mathrm{w}^{+}$; $s v^{\text {spa-pol }}$ (TR-A) or $\mathrm{y}^{+}, \mathrm{sv}^{+}$animals (TR-B).
E

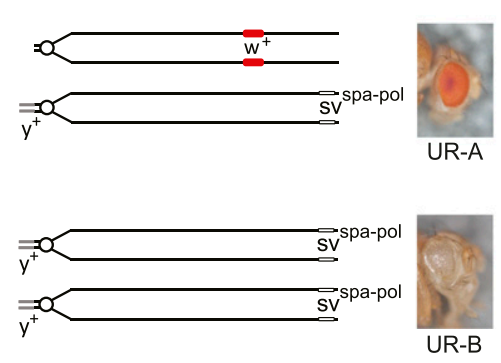

F1 generation:

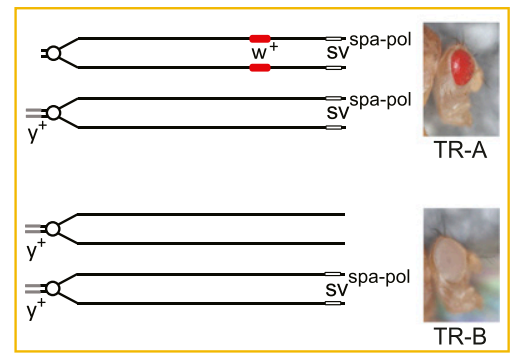

animals were crossed with homozygous yw; $D p(1 ; 4) 1021, y^{+}, s^{\text {spa-pol }}$ flies to visually detect recombination events in the offspring (i.e., the two visible markers co-segregate upon recombination; see Fig 3B and C and see the Materials and Methods section for details). From 8,604 offspring, we recovered 253 putative recombinants (Fig 3D). Of these 253 animals, we further characterized 57 deriving mostly from independent crosses. 21 of the 57 animals turned out to be true germline-transmitted recombinants that were homozygous viable (TRA and TR-B in Fig 3E and Tables S4 and S5). Molecular analysis of the Cas9 target site revealed that 19 of these 21 recombinants did not contain an indel lesion at the CRISPR site (sequence traces with indels are shown in Table S4). Extrapolating from the 21 confirmed recombinants (out of 57) to the 253 putative recombinants (out of 8,604 F1 animals), the frequency of CRISPR-mediated targeted recombination in this experiment is estimated to be $\sim 1.1 \%$. This frequency is at least four orders of magnitude higher than that of the rare spontaneous recombination rate predicted for chromosome 4 (37).

\section{CRISPR-induced DSBs may lead to loss of chromosomal structures}

The remaining 36 (out of 57) putative recombinants recovered from the above experiment were homozygous lethal and exhibited position effect variegation (PEV; Fig 4A and B) in the adult compound eye. PEV in the eye occurs if the mini-white gene $\left(w^{+}\right)$, used as transgene reporter, is juxtaposed to heterochromatic regions via chromosomal rearrangements or translocations $(42,43,44,45)$. In particular, the proximity of the mini-white gene to the heterochromatic telomere regions may lead to PEV (46). We, therefore, reasoned that in animals showing PEV, the Cas9-based editing led to a loss of chromosome structures distal to the DSB and consequently, expression of the miniwhite gene of the CIGAR transgene is variably silenced. Similar events have been reported for $\mathrm{X}$-ray-induced DSBs on the fourth chromosome (47). To assess the presence or absence of the most distal part of the chromosomes showing PEV, we tested CIGAR ${ }^{\text {mCherry,102F,w+/ }}$ chromosomes for complementation of the lethal $S v^{\Delta 122}$ null mutation. Independently recovered $y w ; \quad$ CIGAR ${ }^{\text {mCherry,102F, } w^{+}, \text {PEV }} / D p(1 ; 4) 1021, y^{+}$, $\mathrm{sv}^{\text {spa-pol }}$ animals exhibiting PEV were crossed to $\mathrm{yw} ; \mathrm{Cl}^{\mathrm{D}}, \mathrm{SV}^{\text {spa-pol }} / \mathrm{sv}^{\mathrm{D}^{122}}$ animals. Without exception, the tested PEV chromosomes were unable to complement the $s v^{122}$ mutation, indicating that they must have lost distal parts of chromosome 4 .

\section{Recombination is triggered by, and confined to the site of, Cas9-induced DSBs}

Finally, to exclude that Cas9-induced DSBS merely stimulate nonspecific recombination (NSR) on the fourth chromosome (i.e., 
A

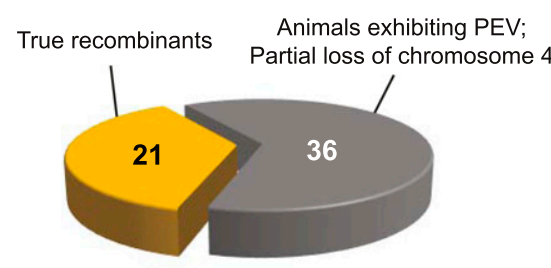

Putative TR analyzed: $n=57$

C
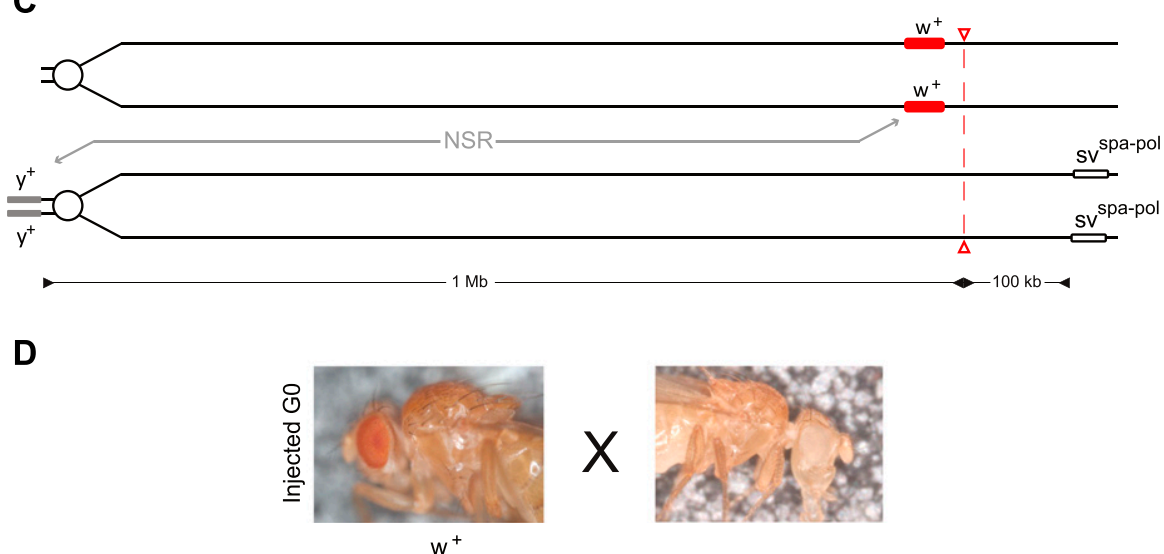

$y w ; \frac{w^{+}}{\operatorname{Dp}(1 ; 4) y^{+}, \text {sv spa-pol }}$
B
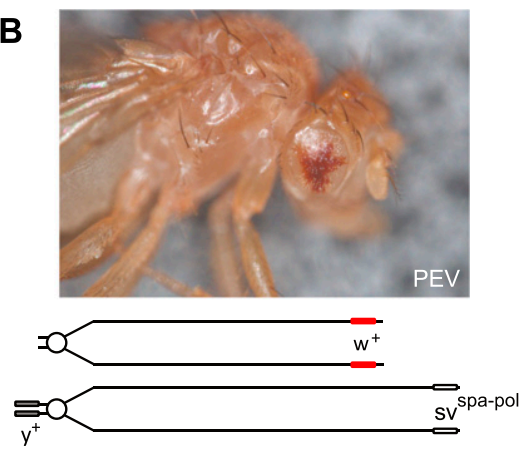

$-100 \mathrm{~kb}-4$
D

E

F1 generation:
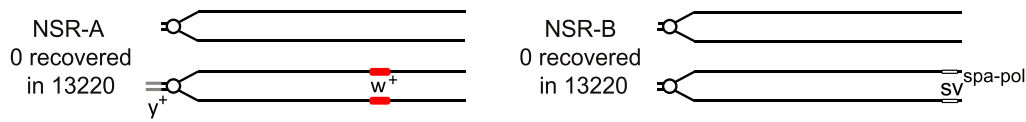

Figure 4. CRISPR-induced DSBs may lead to loss of chromosomal structures.

(A) Of the putative 257 recombinants (see Fig 3D), 57 were characterized in more detail. 21 were true recombinants, whereas 36 animals had a loss of chromosomal structures distal to the CRISPR target site which manifested in PEV, that is, variable expression of the mini-white gene. (B) Fly exhibiting PEV is shown. The genotype of the fly and the loss of chromosomal structures distal to the cut site are shown below the image. Important to note is that only the loss of distal chromosome structures of the $\mathrm{w}^{+}$chromosome can be scored from the TR screen (described in Fig 3). The corresponding loss of $s v^{\text {spa-pol }}$ on the $D p(1 ; 4) 1021, y^{+}$ chromosome is phenotypically identical to UR-B (Fig 3E) and will not be recovered from the screen. (C) Control experiment to assess if CRISPR-Cas9-induced DSBs in general enhances the frequency of recombination away from the Cas9 cut site (dashed line and red open arrowheads). (D) Cas9 RNP-injected animals (same as in Fig 3B) are backcrossed to animals with the genotype yw to be able to visually score NSR events between $\mathrm{w}^{+}$and $y^{+}$. (E) No NSR between $y^{+}$and $w^{+}$marker (NSR-A and NSR-B) were recovered amongst 13,220 animals screened (see text and the Materials and Methods section for more details). not at the Cas9 target site), we repeated the experiment using the same experimental conditions, but assessed recombination between a $y^{+}$marker on the short left arm of chromosome 4 and the $\mathrm{W}^{+}$-marked $\mathrm{CIGAR}^{\text {mCherry,102F }}$ transgene inserted at $102 \mathrm{~F}$ of the right arm (Fig 4C-E, NSR; see the Materials and Methods section for more experimental details). Embryos of the genotype yw; CIGAR ${ }^{\text {mCherry, 102F, } w^{+}}$/ $D p(1 ; 4) 1021, \mathrm{y}^{+}, \mathrm{sv}^{\text {spa-pol }}$ were injected with RNPs containing Cas9 protein complexed with in vitro-translated sgRNA-3. The RNPs induce DSBS $3^{\prime}$ of toy located distal of CIGAR ${ }^{\text {mCherry, } 102 F}$ but should not have any influence on the recombination between the $y^{+}$and the $\mathrm{w}^{+}$ markers. G0 animals were crossed with yw animals to score recombination events between the $y^{+}$and the $w^{+}$marker (Fig 4D). We screened more than 13,000 F1 animals but did not observe a single recombination event between the markers (NSR-A or NSR-B in Fig 4E, see the Materials and Methods section for details). These results are consistent with the notion that no spontaneous recombination occurs between the fourth chromosomes and that the recombination observed in the previous experiments above (Fig 3, TR) were triggered by, and confined to the site of, CRISPR-induced DSBS.

In summary, our experiments involving chromosome 4 revealed that germ-line-transmitted recombinants can be recovered at frequencies ranging from about $1.1 \%$ (recombination between visible markers) to $26 \%$ (recombination between CIGAR constructs; see the Materials and Methods section). These percentages represent the number of recombinants recovered from the total number of animals analyzed. When only the detected Cas9mediated events are taken into consideration, the frequency of recombination was even $39 \%$.

\section{Discussion}

Our results show that a substantial amount of CRISPR-Cas9induced DSBs result in exchanges between homologous chromosome arms. Importantly, the recombination events we see occur in multiple experimental settings and not only under specific conditions. In this context, it is important to point out that recombination events observed in experiments where Cas9 has been provided as recombinant protein exclusively occurred in mitotic and not in meiotic cells: a study by Burger and colleagues shows that fluorescently labeled Cas9 RNP complexes are detectable until about $18 \mathrm{~h}$ after injection (40). In Drosophila, however, the first meiotic divisions occur at much later timepoints (i.e., in males in the 
third instar larvae, about 3-4 d after injection and in females at early pupal stages (48)), likely excluding residual activity of Cas9 RNPS.

In experiments where Cas9 protein and the sgRNAs are provided via transgenes $(49,50)$, Cas9 expression is driven by the nanos (nos) promoter and the Cas9 transgene contains the nos 3'UTR recapitulating germline-specific nos expression, transcript localization, and translational control $(49,51,52)$. Hence, Cas9 is maternally provided to the offspring and likely not expressed zygotically during embryogenesis (53). In females, Nos is available during mitotic divisions in the germline stem cells as well as in those that later on form the 16-cell cyst in the germline. It should not be present in the growing oocyte where meiosis would occur (53). In the male germline, nos expression seems to be essential during spermatogenesis (54). Loss of nos expression leads to various phenotypes with strongest effects on the number of primary spermatocytes that are created through mitotic divisions. Together, these arguments suggest that also in case the CRISPR reagents are provided via transgenes, recombination events occurred preferentially in mitotic cells. However, because the nos-Cas9 transgene is not inserted into the nos locus, presence of Cas9 protein and extension recombination events in meiotic cells cannot strictly be ruled out.

One reason why CRISPR-induced recombination has largely remained unnoticed in genetic model organisms is that DSBs are frequently repaired without resulting indels and thus cannot be detected by next-generation sequencing, preventing any follow-up validation of broader effects. When detected using genetic and sequencing validation, we noted variable recombination rates in our experiments. We attribute this variability to three experimental aspects. First, the different Cas9 target sites may have different cutting and recombination efficacy $(30,55)$. Second, individual crosses may yield different numbers of progeny harboring CRISPRderived indels (i.e., CRISPRed (G0) animals transmit a variable number of mutant alleles to the next generation, ranging from 0 to $100 \%$ as shown for the recovery of a nonfunctional $y$ allele, see Fig 2 in reference 49). Third, in our experiments, different methods were used to introduce Cas9 and gRNAs, a circumstance that likely contributed to the different frequencies of recombinants recovered (49).

The Cas9-mediated site-specific chromosomal recombination described here opens up a number of new avenues and considerations for genome engineering. We demonstrate that in Drosophila, Cas9-mediated DSBs can be used to generate recombination at a predefined site between chromosomal locations that are in close vicinity. Unlike for site-specific recombination based on Flp $(56,57)$, no recombinase target sites need to be present. Thus, CRISPR-mediated site-specific recombination enables the combination of two known mutations situated in different alleles of one and the same gene into a single (double-)mutant allele. Moreover, it could be used to study permutations of mutant alleles in tightly linked genes such as members of a Hox gene cluster (58). Especially in vertebrates, such as mouse or zebrafish, CRISPR-induced recombination holds promise for a number of applications, including generation of complex mutant alleles in the same locus (59). Also, in Drosophila, Cas9-mediated recombination enables experiments that were previously impossible because of low or absent recombination rates. For example, by providing sgRNAs or Cas9 activity in a tissue-specific manner, it may become possible to generate specific mutant clones for genes located on the fourth chromosome. Finally, targeted Cas9-mediated recombination could potentially be used in epigenetic studies to determine the effects of swapping promoters, including their epigenetic marks, between maternal and paternal genes.

On the other hand, our findings that recombination between homologous chromosome arms may be triggered upon Cas9induced DSBS underscore the need for caution in applying CRISPR-based genetic interventions in animals or humans (60): Unrecognized CRISPR-induced recombination events (i.e., no visible indels at the target site) may lead to loss-of-heterozygosity events, generating cells with unnoticed homozygosity for imprinted genes or recessive mutations located distal to the Cas9 target site, which may have unforeseen consequences.

Moreover, we observed that CRISPR-based engineering can lead to loss of chromosome material distal to the locus of the DSB (Fig 4B). We could observe such events in our system because haplo-four animals (harboring only a single copy of the fourth chromosome) are viable (61). Under most circumstances, and likely in most organisms, such events would lead to the death of the affected cells because of haploinsufficiency. In this context, recent work suggested that Cas9 induces mutations in human cells and mice that are larger than anticipated (62). Therefore, it is imperative to routinely consider broader chromosomal alternations as possible outcome when applying CRISPR technologies in translational medicine.

\section{Materials and Methods}

\section{Plasmid construction}

Unless otherwise noted, the plasmids were constructed by standard molecular cloning methods. When plasmids contain newly synthesized nt sequences via PCR, oligonucleotide synthesis, or mutagenesis, the sequences were verified by DNA sequencing.

\section{pUbiattB}

The Stul-ubiquitin p63E promoter (ubi)-EcoRl fragment from pCaSpR3-Up2-RX polyA was subcloned into a pBluescript ( $p B S$ ) vector between the ECoRI and Xhol sites using blunt-end ligation. The EcoRI-ubi-Acc65I fragment from the resulting plasmid was subcloned into pEPattB (63) using the EcoRI and Acc65I sites.

\section{pUbiattB-CIGAR ${ }^{\text {eGFP }}$}

To create the pUbiattB-CIGAR ${ }^{\text {eGFP }}$ reporter, the shifter sequence (containing optimized translational START codons covering all three frames upstream of a unique 20-nt CRISPR target sequence), a unique gRNA target sequence (referred to as sgRNA-1), the linker sequence, and the eGFP gene were designed (as shown below), synthesized by GenScript, and delivered ligated into the pUC57-Kan vector ( $P C I G A R-D O)$.

The $P C I G A R-D O$ insert:

5'-KpnI_CAACATGGTGCAACATGGTGCAACATGGTGCGGCGACAGCAGA ACGTAGCGGGACGATAGGCTGCAGATCCTTGGCGCGCCTTCAGGAGGCGGT- 
GCTACTGCTGGCGCTGGTGGAGCCGGTGGACCTGCGGGGTTAATTGTGAGCAAGGGCGAGGAGCTGTTCACCGGGGTGGTGCCCATCCTGGTCGAGCTGGACGGCGACGTAAACGGCCATAAGTTCAGCGTGTCCGGCGAGGGCGAGGGCGATGCCACCTACGGCAAGCTGACCCTGAAGTTCATCTGCACCACCGGCAAGCTGCCCGTGCCCTGGCCCACCCTTCGTGACCACCCTGACCTACGGCGTGCAGTGCTTCAGCCGCTACCCCGACCACATGAAGCAGCACGACTTCTTCAAGTCCGCCATGCCCGAAGGCTACGTCCAGGAGCGCACCATCTTCTTCAAGGACGACGGCAACTACAAGACCCGCGCCGAGGTGAAGTTCGAGGGCGACACCCTGGTGAACCGCATCGAGCTGAAGGGCATCGACTTCAAGGAGGACGGCAACATCCTGGGGCACAAGCTGGAGTACAACTACAACAGCCACAACGTCTATATCATGGCCGACAAGCAGAAGAACGGCATCAAGGTGAACTTCAAGATCCGCCACAACATCGAGGACGGCAGCGTGCAGCTCGCCGACCACTACCAGCAGAACACCCCCATCGGCGACGGCCCCGGTGCTGCTGCCCGACAACCACTACCTGAGCACCCAGTCCGCCCTGAGCAAAGACCCCAACGAGAAGCGCGATCACATGGTCCTGCTGGAGTTCGTGACCGCCGCCGGGATCACTCTCGGCATGGACGAGCTGTACAAGTAAGAATTC_ECORI-3'.

The synthetic sequence was excised with $K p n I$ and EcoRl and ligated into pre-double-digested pKB342 vector in line with a tubulin $3^{\prime}$ trailer, transformed, and purified, resulting in PKB342_Cl$G A R$. To remove the CIGAR sequence including the tubulin trailer, PKB342_CIGAR was then digested with Kpnl and Xbal, and the fragment was ligated into a Kpnl- and Xbal-digested pUbiattB vector containing an ubiquitin-p63E promoter (see above). This resulted in the final product referred to as pUbiattB-CIGAR ${ }^{\text {eGFP. }}$.

\section{pUbiattB-CIGAR ${ }^{\text {mCherry }}$}

The same sequential digestions and ligation as for the making of the pUbiattB-CIGAR ${ }^{\text {EGFP }}$ were used for the construction of the pUbiattBCIGAR ${ }^{\text {mCherry }}$. The only difference was in the design of the target site; the gRNA (sgRNA-2) has a different unique target sequence and contains the mCherry gene as the fluorescent marker. The insert ligated into the pUC57-Kan vector was ordered from GenScript.

The CIGAR ${ }^{\text {mCherry }}$ insert:

5'-KpnI_CAACATGGTGCAACATGGTGCAACATGGTGCCCCGAGACAAGCACCTGACGGGACGATAGGCTGCAGATCCTTGGCGCGCCTTCAGGAGGCGGTGCTACTGCTGGCGCTGGTGGAGCCGGTGGACCTGCGGGGTTAATTGTGAGCAAGGGCGAGGAGGACAACATGGCCATCATCAAGGAGTTCATGCGCTTTAAGGTGCACATGGAGGGCTCCGTGAACGGCCACGAGTTCGAGATCGAGGGCGAGGGCGAGGGCCGCCCCTACGAGGGCACCCAGACCGCCAAGCTGAAGGTGACCAAGGGCGGCCCCCTGCCCTTCGCCTGGGACATCCTGTCCCCTCAGTTCATGTACGGCTCCAAGGCCTACGTGAAGCACCCCGCCGACATCCCCGACTACTTGAAGCTGTCCTTCCCCGAGGGCTTCAAGTGGGAGCGCGTGATGAACTTCGAGGACGGCGGCGTGGTGACCGTGACCCAGGACTCCTCCCTGCAGGACGGCGAGTTCATCTACAAGGTGAAGCTGCGCGGCACCAACTTCCCCTCCGACGGCCCCGTAATGCAGAAGAAGACCATGGGCTGGGAGGCCTCCTCCGAGCGGATGTACCCCGAGGACGGCGCCCTGAAGGGCGAGATCAAGCAGAGGCTGAAGCTGAAGGACGGCGGCCACTACGACGCCGAGGTCAAGACCACCTACAAGGCCAAGAAGCCCGTGCAGCTGCCCGGCGCCTACAACGTCAACATCAAGCTGGACATCACCTCCCACAACGAGGACTACACCATCGTGGAACAGTACGAGCGCGCCGAGGGCCGCCACTCCACCGGCGGCATGGACGAGCTGTACAAGTAA_ECORI-3'.

\section{U6:3-sgRNA ${ }^{\text {CIGAR(1,2) }}$ (pCFD5-F1)}

The pCFD5 vector was a gift from Fillip Port (\#73914; Addgene) (50). pCFD5 is the main backbone containing the tRNA assembly prepared to insert multiple gRNAs. For our purposes, sgRNA-1 and sgRNA-2 were inserted via Gibson cloning into the pCFD5 vector (named pCFD5-F1) following the protocol described in the supplementary methods of pCFD5 cloning protocol.

pCFD5 internal tRNA multi-gRNA Scaffold:

5'-GTCGGGGCTTTGAGTGTGTGTAGACATCAAGCATCGGTGGTTCAGTGGTAGAATGCTCGCCTGCCACGCGGGCGGCCCGGGTTCGATTCCCGGCCGATGCAGGGTCTTCGTTTTAGAGCTAGAAATAGCAAGTTAAAATAAGGCTAGTCCGTTATCAACTTGAAAAAGTGGCACCGAGTCGGTGCAACAAAGCACCAGTGGTCTAGTGGTAGAATAGTACCCTGCCACGGTACAGACCCGGGTTCGATTCCCGGCTGGTGCAGAAGACCTGTTTTAGAGCTAGAAATAGCAAGTTAAAATAAGGCTAGTCCGTTATCAACTTGAAAAAGTGGCACCGAGTCGGTGCTTTTTT-3'.

Through the process of Gibson cloning, both our sgRNA-1 and sgRNA-2 were inserted into the PCFD5 vector using primer (5'GCGGCCCGGGTTCGATTCCCGGCCGATGCACGGCGACAGCAGAACGTAGCGTTTTAGAGCTAGAAATAGCAAG-3') for sgRNA-1 and (5'-ATTTTAACTTGCTATTTCTAGCTCTAAAACGTCAGGTGCTTGTCTCGGGGTGCACCAGCCGGGAATCGAACCC-3') for SgRNA-2.

\section{IVT of sgRNA-3}

IVT of sgRNAs were performed as described in reference 40. For IVT, we used MEGAscript (AM1334), and for the purification of the IVT products, we used Purification: MEGAclear (AM1908).

IVT oligo used specific for sgRNA-3:

5'-GAAATTAATACGACTCACTATAGGCTGTTGATAAGCACGCAATCGTTT-

TAGAGCTAGAAATAGC-3'.

IVT oligo used sgRNA-R used for template PCR:

5'-AAAAGCACCGACTCGGTGCCACTTTTTCAAGTTGATAACGGACTAGCCTTATTTTAACTTGCTATTTCTAGCTCTAAAAC-3'.

Complementary sequences of the specific sgRNA primer and the sgRNA-R are shown in bold letters.

\section{Cas9/sgRNA RNP injections}

The concentration of SpCas9 injected was about $800 \mathrm{ng} / \mu \mathrm{l}$ SpCas9 (final concentration) and about $300 \mathrm{ng} / \mu \mathrm{l}$ of sgRNA (IVT; final concentration after purification). This corresponds roughly to a 1:2 ratio (SpCas9: sgRNA-3). SpCas9 has about $5 \times$ the molecular weight of the sgRNA.

The injection mix is prepared as follows (total volume of $10 \mu \mathrm{l}$ ): add sgRNA, dd $\mathrm{H}_{2} \mathrm{O}$, and $10 \times$ incubation buffer NEB and mix thoroughly. Gently add the SpCas9 and mix again thoroughly by pipetting up and down. It is mandatory to add SpCas9 as the last ingredient because low salt concentrations may cause SpCas9 to precipitate (40).

$\mathrm{X} \mu \mathrm{l}$ sgRNA (IVT; to a final concentration of $320 \mathrm{ng} / \mu \mathrm{l}$; corresponds to $1: 2$ ratio SpCas9: sgRNA).

$1 \mu \mathrm{l} 10 \times$ NEB incubation buffer.

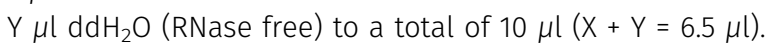

$2.5 \mu \mathrm{l}$ SpCas9 (EnGen Cas9 NLS, NEB \#M0646T; $3.22 \mu \mathrm{g}$; final concentration $0.8 \mu \mathrm{g} / \mu \mathrm{l})$.

Mix gently and incubate the mix at $37^{\circ} \mathrm{C}$ for $2 \mathrm{~min}$.

Load the mix onto a column (Ultrafree-MC-HV $0.45 \mu \mathrm{m}$ [Ref: UFC30HV00]).

Spin for 1 min in a table-top centrifuge @14,000g.

Reincubate the flow-through at $37^{\circ} \mathrm{C}$ for $2 \mathrm{~min}$.

Let the mix equilibrate at RT for $\sim 30$ min before injection. Never put the mix back on ice. 


\section{Fly genetics}

Crosses were done at $25^{\circ} \mathrm{C}$. Unless noted otherwise, fly lines were obtained from the Bloomington Drosophila Stock Center (see the Acknowledgments section).

Transgenic CIGAR fly lines (integration into ZH-attP 5D [X chromosome] or ZH-attP 102F [fourth chromosome]) were generated by phic31 integrase-mediated transgenesis $(51,64)$. Individual strains were confirmed to carry the correct shifter sequence by sequencing the PCR product of the shifter sequence using primers CIGAR-fwd: CAACAAAGTTGGCGTCGATA and CIGAR ${ }^{\text {EGFP }}$-rev: GAACTTCAGGGTCAGCTTGC

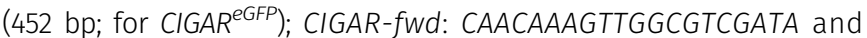
CIGAR ${ }^{\text {mCherry }}$-rev: AAGCGCATGAACTCCTTGATG (367 bp; for CIGAR ${ }^{\text {mCherry }}$ ), respectively. $P C R$ settings were as follows: $95^{\circ} \mathrm{C}, 5 \mathrm{~min} ; 35$ cycles of $95^{\circ} \mathrm{C}$, $25 \mathrm{~s} ; 60^{\circ} \mathrm{C}, 25 \mathrm{~s}$; and $72^{\circ} \mathrm{C}, 30 \mathrm{~s}$ ); final elongation of $72^{\circ} \mathrm{C}, 10 \mathrm{~s}$. The same $P C R$ setting was used to analyze the shifter sequence of the CIGAR reporters by single fly PCR.

The pCFD5-F1 containing U6:3-sgRNA ${ }^{\operatorname{CIGAR}(1,2)}$ was inserted into the attP 40 site (\#25709; Bloomington).

\section{Fly images}

The images were taken on Axio Zoom V16 (Zeiss) and were processed in Adobe Photoshop or Adobe Illustrator.

\section{CRISPR-Cas9-induced recombination on the fourth chromosome (more detailed description).}

To determine if we could also induce CRISPR/Cas9-mediated recombination on the fourth chromosome, we first generated flies harboring either a CIGAR ${ }^{\text {EFP }}$ or CIGAR ${ }^{\text {mCherry }}$ construct on the fourth chromosome at position $102 F$ (51). These animals are referred to as CIGAR ${ }^{\text {EFPP, 102F, } w_{+}^{+}}$and CIGAR ${ }^{\text {mCherry, 102F, } w^{+}}$, respectively. Activation of the reporters was achieved with the previously used tRNA spaced sgRNA-1,2 tandem array (U6:3-sgRNA $\left.{ }^{\operatorname{CIGAR}(1,2)}\right)$. To test if CRISPRCas9-mediated DSBS also result in recombination, the fourth

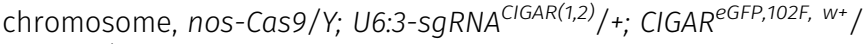
CIGAR ${ }^{\text {mCherry,102F, } w^{+}}$G0 males were crossed to yw females (or nosCas9/+; U6:3-sgRNA ${ }^{\text {CIGAR(1,2) } /+; ~ C I G A R}$ GFP,102F, w+ $_{\text {CIGAR }}^{\text {mCherry, 102F, w+ }}$ G0 females to $y w$ males). The offspring of such crosses were first scored at the larval stage to identify animals with either an activated $\mathrm{CIGAR}{ }^{\text {EGFP,102F }}$ or CIGAR ${ }^{\text {mCherry,102F }}$ reporter. This preselection was made to ascertain that DSBS occurred in both constructs enhancing the likelihood of detecting recombination events on the fourth chromosome in case they occur. 17 vials (crosses from 12 G0 males and 5 G0 females) containing GFP and mCherry-positive larvae were selected for further analysis. A total of $172 \mathrm{y}, \mathrm{w}^{+} \mathrm{F} 1$ animals harboring either a CIGAR ${ }^{\text {EGF, 102F }}$ or CIGAR ${ }^{\text {mCherry, 102F }}$ reporter but lacking the sgRNA plasmid U6:pCFD5 (to avoid mosaic flies) from 13 of these crosses were randomly picked right after hatching. Single fly PCR of the target as well as part of the fluorophore region for the CIGAR reporters was performed for these 172 animals (Tables S2 and S3). PCR products and readable sequences were obtained in 156 cases.

For the recombination experiments using the CIGAR transgenes on chromosome 4, we estimate the recombination frequency to be
26\%: 41 recombinants identified/ 156 flies analyzed $\times 100$ (see Fig $2 C$ for numbers).

\section{CRISPR-induced recombination between two phenotypic markers (TR in Fig 3)}

To confirm that indeed recombination between sister chromatids occurs after CRISPR-Cas9-induced DSBS, we tested if we could induce recombination between two visible markers that are separated by about $100 \mathrm{~kb}$ (Figs $3 \mathrm{~A}$ and S3). The markers were $\mathrm{W}^{+}$-marked CIGAR ${ }^{\text {mCherry, } 102 \mathrm{~F}, \mathrm{w}^{+}}$at $102 \mathrm{~F}$ and the recessive viable mutation $s v^{\text {spa-pol }}$ (a mutation in the eye-specific enhancer of the Drosophila Pax 2 gene) located downstream of the $\mathrm{w}^{+}$marker near the tip of chromosome 4 (65). The Cas9 target site was selected in the $3^{\prime}$ UTR of the toy gene residing about $18 \mathrm{~kb}$ downstream of the CIGAR ${ }^{\text {mCherry,102F, } w^{+}}$transgene insertion site (Fig S3). The rough-eye phenotype of $s v^{\text {spa-pol }}$ is reliably scored (100\% penetrance) and is visible if the mutation is homozygous or over a null allele of sv such as sv ${ }^{\Delta 122}$ (Sabarinadh Chilaka, Michael Daube, Erich Frei, and Markus Noll, in preparation).

$y w ; C^{2}$ CIGAR ${ }^{\text {mCherry,102F, }} w^{+} / D p(1 ; 4) 1021, y^{+}, \quad s v^{\text {spa-pol }}$ embryos were injected with recombinant Cas 9 protein complexed with in vitro-translated sgRNA-3 targeting the 3'UTR of the toy gene. From the eclosing $\mathrm{G} 0$ animals, a total of 135 crosses were set up. Either five G0 females ( 29 crosses) or single G0 males (106 crosses) were

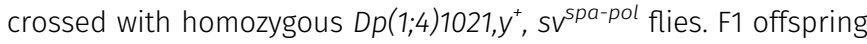
were either $y w$; CIGAR ${ }^{\text {mCherry,102F, }} w^{+} / D p(1 ; 4) 1021, y^{+}$, sv $^{\text {spa-pol }}$ (UR-A; phenotypically $\left.\mathrm{w}^{+}, \mathrm{y}^{+} \mathrm{Sv}^{+}\right)$or homozygous $D p(1 ; 4) 1021, \mathrm{y}^{+}, s v^{\text {spa-pol }}$ animals (UR-B; phenotypically $w, y^{+}, s v^{\text {spa-pol) }}$ (Fig 3E). Recombination events would be phenotypically distinct (Fig 3E; TR-A or TR-B): TR-A is yw; CIGAR ${ }^{\text {mCherry,102F, w+ }}$, sv spa-pol $^{\text {D }}$ Dp (1;4)1021, $y^{+}$, sv $^{\text {spa-pol }}$ and would be scored as having rough, red eyes $\left(w^{+}, y^{+} s v^{s p a-p o l}\right)$. The second recombination event (TR-B) that could occur would be yw; Dp (1;4)1021, $y^{+}$, $s v^{+} / D p(1 ; 4) 1021, y^{+}, s v^{s p a-p o l}$ flies having white and smooth eyes $\left(w, y^{+}\right.$ $\left.\mathrm{SV}^{+}\right)$. From a total of 8,604 scored $\mathrm{F} 1$ animals, 253 putative recombinant flies were recovered: 216 had rough red eyes (2.5\%, from 56 independent $\mathrm{GO}$ crosses) and 37 animals had white and smooth eyes (five independent $\mathrm{G} 0$ crosses). Notably, in one cross, $31 \mathrm{w}^{-}, \mathrm{y}^{+} \mathrm{Sv}^{+}$ animals were present in one tube corresponding to about $25-30 \%$ of the offspring. 70 of the 253 putative recombinants (we picked mostly males) were backcrossed to homozygous $D p(1 ; 4) 1021, y^{+}, s v^{\text {spa-pol }}$ flies to establish stocks. 61 of the 70 putative recombinants were recovered from independent crosses ( $56 w^{+}, s v^{s p a-p o l}$ and $\left.5 w, y^{+}, s v^{+}\right) .13$ of the 70 crosses remained without offspring. Most importantly, from 21 putative recombinants, we could generate homozygous viable lines. 17 of the 21 lines showed the $y, w^{+}, s v^{\text {spa-pol }}$ phenotype, whereas four lines were $w$, $\mathrm{y}^{+}, \mathrm{sv}^{+}$marking all 21 lines as true recombinants. The remaining 36 putative recombinants were homozygous lethal (for more information on these 36 lines see "Cas9-induced DSBs may lead to loss of chromosomal structures" below).

We then investigated these animals by PCR specific for the CRISPR target site. Sequence analysis revealed that with the exception of two animals all CRISPR sites were without any indel (i.e., had a wild-type sequence; Tables S4 and S5). Interestingly, two recombinants from the same cross (G0 male) showed the same CRISPR mark (6-bp deletion). However, they had the complementary phenotype: Whereas one fly showed the $y, w^{+}, s v^{\text {spa-pol }}$ 
phenotype, the other was phenotypically $w, y^{+}, s v^{+}$, indicating that we may have recovered both chromosomes from the same recombination event. A third recombined animal from the same cross showed no CRISPR mark and thus can be counted as independent recombination event.

\section{Recombination experiment between two phenotypic markers (NSR in Fig 4)}

To assess if CRISPR-Cas9-induced DSBs in general would enhance the frequency of recombination away from the Cas9 target site, we repeated the experiment and assessed the recombination frequency between a $y^{+}$marker on the short, left arm of chromosome 4 and the $\mathrm{W}^{+}$-marked CIGAR ${ }^{\text {mCherry, } 102 \mathrm{~F}}$ inserted at $102 \mathrm{~F}$ (see NSR in Fig 4C). To use the same experimental conditions and to exclude that the addition of active Cas9 would also induce recombination elsewhere on the fourth chromosome, embryos of the genotype (yw; CIGAR ${ }^{\text {mCherry, } 102 F, w^{+}} / D p(1 ; 4) 1021, y^{+}$, sv $\left.^{\text {spa-pol }}\right)$ were injected with Cas9 protein complexed with in vitro-translated SgRNA-3. This again would induce DSBs $3^{\prime}$ of toy located distal of CIGAR ${ }^{\text {mCherry,102F }}$ but should not have any influence on the recombination between the $\mathrm{y}^{+}$and the $\mathrm{w}^{+}$markers. G0 animals were this time crossed with $y w$ animals to be able to score crossover events between the $y^{+}$and the $w^{+}$marker (Fig 4D). As expected, F1 animals were phenotypically either $y w^{+}$or $y^{+} w$ because the two markers normally segregate. However, 11 phenotypically $y^{+}, w^{+}$flies were recovered from a total of 13,220 analyzed offspring. To assess if the $y^{+}, w^{+}$recovered animals were indeed spontaneous recombinants, or represented nondisjunction events (i.e., triplo-4 animals which are viable), we backcrossed such animals again against yw flies. Spontaneous recombination between $y^{+}$and $w^{+}$could be excluded as the abovementioned backcross would have only revealed phenotypically $\mathrm{y}^{+} \mathrm{w}^{+}$or $\mathrm{yw}$ flies. Instead, nondisjunction could be confirmed in all 11 cases because the backcross revealed phenotypically $y^{+} w^{+}, y w, y^{+} w$, and $y w^{+}$flies. We determined the non-disjunction rate for the fourth chromosome to be 1 in 1,200, which is similar to the one observed for the $X$ chromosome (66).

\section{Supplementary Information}

Supplementary Information is available at https://doi.org/10.26508/lsa. 201800267.

\section{Acknowledgements}

We thank Julia Compart, Angelika Dannert, Madlen Mueller, and Andri Lansel for technical help, Giuseppe Saccone, Daniel Bopp, Alessandro A Sartori, Damian Brunner, Darren Gilmour, Hugo Stocker, Olivier Urwyler, Ernst Hafen, Hirokazu Okada, Markus Noll, Christian Lehner, and Erich Frei for scientific discussions; Fillip Port for reagents; Johannes Bischoff for the ZH attP 5D line; Markus Noll for the $y w ; s v^{\Delta 122} / \mathrm{Ci}^{\mathrm{D}}, s v^{\text {spa-pol }}$ fly stock; and Martin Jinek and Giuseppe Saccone for recombinant Cas9 protein for testing. This work was supported by the Swiss National Science Foundation (SNF), grant number 310030B_173331 (K Basler), Swiss National Science Foundation, grant number 170623 (C Mosimann), Swiss Bridge Foundation (A Burger), SNF consecutive grants 31003A_162557 and 31003A_182532, and by the SystemsX.ch grant 51RT-0_145725 (to Ernst Hafen; ETH Zurich).

\section{Author Contributions}

E Brunner: conceptualization, data curation, formal analysis, supervision, validation, investigation, visualization, methodology, project administration, and writing-original draft, review, and editing.

$\mathrm{R}$ Yagi: conceptualization, resources, formal analysis, validation, investigation, visualization, methodology, and writing-original draft, review, and editing.

M Debrunner: validation, investigation, methodology, and writing-review and editing.

D Beck-Schneider: investigation, methodology, and writing-original draft, review, and editing.

A Burger: resources, methodology, and writing-review and editing. E Escher: investigation and methodology.

C Mosimann: resources, funding acquisition, methodology, and writing-review and editing.

G Hausmann: funding acquisition and writing-review and editing K Basler: conceptualization, resources, supervision, funding acquisition, project administration, and writing-review and editing.

\section{Conflict of Interest Statement}

The authors declare that they have no conflict of interest.

\section{References}

1. Jinek M, Chylinski K, Fonfara I, Hauer M, Doudna JA, Charpentier E (2012) A programmable dual-RNA-guided DNA endonuclease in adaptive bacterial immunity. Science 337: 816-821. doi:10.1126/science.1225829

2. Doudna JA, Charpentier E (2014) Genome editing. The new frontier of genome engineering with CRISPR-Cas9. Science 346: 1258096. doi:10.1126/science.1258096

3. Hsu PD, Lander ES, Zhang F (2014) Development and applications of CRISPR-Cas9 for genome engineering. Cell 157: 1262-1278. doi:10.1016/ j.cell.2014.05.010

4. Bosley KS, Botchan M, Bredenoord AL, Carroll D, Charo RA, Charpentier E, Cohen R, Corn J, Doudna J, Feng G, et al (2015) CRISPR germline engineering: The community speaks. Nat Biotechnol 33: 478-486. doi:10.1038/nbt.3227

5. Sternberg SH, Doudna JA (2015) Expanding the biologist's toolkit with CRISPR-Cas9. Mol Cell 58: 568-574. doi:10.1016/j.molcel.2015.02.032

6. Chavez A, Tuttle M, Pruitt BW, Ewen-Campen B, Chari R, Ter-Ovanesyan D, Haque SJ, Cecchi RJ, Kowal EJK, Buchthal J, et al (2016) Comparison of Cas9 activators in multiple species. Nat Methods 13: 563-567. doi:10.1038/nmeth.3871

7. Ewen-Campen B, Mohr SE, Hu Y, Perrimon N (2017) Accessing the phenotype gap: Enabling systematic investigation of paralog functional complexity with CRISPR. Dev Cell 43: 6-9. doi:10.1016/j.devcel.2017.09.020

8. Sadhu MJ, Bloom JS, Day L, Siegel JJ, Kosuri S, Kruglyak L (2018) Highly parallel genome variant engineering with CRISPR-Cas9. Nat Genet 50: 510-514. doi:10.1038/s41588-018-0087-y

9. Sander JD, Joung JK (2014) CRISPR-Cas systems for editing, regulating and targeting genomes. Nat Biotechnol 32: 347-355. doi:10.1038/nbt.2842 
10. Barrangou R, Doudna JA (2016) Applications of CRISPR technologies in research and beyond. Nat Biotechnol 34: 933. doi:10.1038/nbt.3659

11. Adli M (2018) The CRISPR tool kit for genome editing and beyond. Nat Commun 9: 1911. doi:10.1038/s41467-018-04252-2

12. Esvelt KM, Mali P, Braff JL, Moosburner M, Yaung SJ, Church GM (2013) Orthogonal Cas9 proteins for RNA-guided gene regulation and editing. Nat Methods 10: 1116-1121. doi:10.1038/nmeth.2681

13. Bosch PS, Ziukaite R, Alexandre C, Basler K, Vincent JP (2017) Dpp controls growth and patterning in Drosophila wing precursors through distinct modes of action. Elife 6: e22546. doi:10.7554/elife.22546

14. Lieber MR (2010) The mechanism of double-strand DNA break repair by the nonhomologous DNA end joining pathway. Annu Rev Biochem 79: 181-211. doi:10.1146/annurev.biochem.052308.093131

15. Guirouilh-Barbat J, Huck S, Bertrand P, Pirzio L, Desmaze C, Sabatier L, Lopez BS (2004) Impact of the KU80 pathway on NHEJ-induced genome rearrangements in mammalian cells. Mol Cell 14: 611-623. doi:10.1016/ j.molcel.2004.05.008

16. Symington LS, Gautier J (2011) Double-strand break end resection and repair pathway choice. Annu Rev Genet 45: 247-271. doi:10.1146/annurevgenet-110410-132435

17. Bétermier M, Bertrand P, Lopez BS (2014) Is non-homologous endjoining really an inherently error-prone process? PLOS Genet 10: e1004086. doi:10.1371/journal.pgen.1004086

18. Pannunzio NR, Watanabe G, Lieber MR (2018) Nonhomologous DNA endjoining for repair of DNA double-strand breaks. I Biol Chem 293: 10512-10523. doi:10.1074/jbc.tm117.000374

19. Geisinger JM, Turan S, Hernandez S, Spector LP, Calos MP (2016) In vivo blunt-end cloning through CRISPR/Cas9-facilitated non-homologous end-joining. Nucleic Acids Res 44: e76. doi:10.1093/nar/gkv1542

20. Ghezraoui H, Piganeau M, Renouf B, Renaud J-B, Sallmyr A, Ruis B, Oh S, Tomkinson AE, Hendrickson EA, Giovannangeli C, et al (2014) Chromosomal translocations in human cells are generated by canonical nonhomologous end-joining. Mol Cell 55: 829-842. doi:10.1016/j.molcel.2014.08.002

21. Zheng Q, Cai X, Tan MH, Schaffert S, Arnold CP, Gong X, Chen C-Z, Huang S (2014) Precise gene deletion and replacement using the CRISPR/Cas9 system in human cells. BioTechniques 57: 115-124. doi:10.2144/000114196

22. Guo T, Feng YL, Xiao JJ, Liu Q, Sun XN, Xiang JF, Kong N, Liu SC, Chen GQ, Wang $Y$, et al (2018) Harnessing accurate non-homologous end joining for efficient precise deletion in CRISPR/Cas9-mediated genome editing. Genome Biol 19: 170. doi:10.1186/s13059-018-1518-x

23. Choi PS, Meyerson M (2014) Targeted genomic rearrangements using CRISPR/Cas technology. Nat Commun 5: 3728. doi:10.1038/ncomms4728

24. Torres R, Martin MC, Garcia A, Cigudosa JC, Ramirez JC, Rodriguez-Perales S (2014) Engineering human tumour-associated chromosomal translocations with the RNA-guided CRISPR-Cas9 system. Nat Commun 5: 3964. doi:10.1038/ncomms4964

25. Maddalo D, Manchado E, Concepcion CP, Bonetti C, Vidigal JA, Han YC, Ogrodowski P, Crippa A, Rekhtman N, de Stanchina E, et al (2014) In vivo engineering of oncogenic chromosomal rearrangements with the CRISPR/Cas9 system. Nature 516: 423-427. doi:10.1038/nature13902

26. Sadhu MJ, Bloom JS, Day L, Kruglyak L (2016) CRISPR-directed mitotic recombination enables genetic mapping without crosses. Science 352: 1113-1116. doi:10.1126/science.aaf5124

27. Heinze SD, Kohlbrenner T, Ippolito D, Meccariello A, Burger A, Mosimann C, Saccone G, Bopp D (2017) CRISPR-Cas9 targeted disruption of the yellow ortholog in the housefly identifies the brown body locus. Sci Rep 7: 4582. doi:10.1038/s41598-017-04686-6

28. Filler Hayut S, Melamed Bessudo C, Levy AA (2017) Targeted recombination between homologous chromosomes for precise breeding in tomato. Nat Commun 8: 15605. doi:10.1038/ncomms15605
29. Bassett AR, Tibbit C, Ponting CP, Liu JL (2013) Highly efficient targeted mutagenesis of Drosophila with the CRISPR/Cas9 system. Cell Rep 4: 220-228. doi:10.1016/j.celrep.2013.06.020

30. Ren X, Yang Z, Xu J, Sun J, Mao D, Hu Y, Yang SJ, Qiao HH, Wang X, Hu Q, et al (2014) Enhanced specificity and efficiency of the CRISPR/Cas9 system with optimized sgRNA parameters in Drosophila. Cell Rep 9: 1151-1162. doi:10.1016/j.celrep.2014.09.044

31. Lee HS, Simon JA, Lis JT (1988) Structure and expression of ubiquitin genes of Drosophila melanogaster. Mol Cell Biol 8: 4727-4735. doi:10.1128/mcb.8.11.4727

32. Jankovics F, Brunner D (2006) Transiently reorganized microtubules are essential for zippering during dorsal closure in Drosophila melanogaster. Dev Cell 11: 375-385. doi:10.1016/j.devcel.2006.07.014

33. Cavener DR (1987) Comparison of the consensus sequence flanking translational start sites in Drosophila and vertebrates. Nucleic Acids Res 15: 1353-1361. doi:10.1093/nar/15.4.1353

34. Kozak M (1986) Point mutations define a sequence flanking the AUG initiator codon that modulates translation by eukaryotic ribosomes. Cell 44: 283-292. doi:10.1016/0092-8674(86)90762-2

35. Bopp D, Schütt C, Puro J, Huang H, Nöthiger R (1999) Recombination and disjunction in female germ cells of Drosophila depend on the germline activity of the gene sex-lethal. Dev Camb Engl 126: 5785-5794.

36. Ashburner M (2011) Drosophila: A Laboratory Handbook. Cold Spring Harbor, NY: Cold Spring Harbor Laboratory Press.

37. Hartmann MA, Sekelsky J (2017) The absence of crossovers on chromosome 4 in Drosophila melanogaster: Imperfection or interesting exception? Fly (Austin) 11: 253-259. doi:10.1080/19336934.2017.1321181

38. Sandler L, Szauter P (1978) The effect of recombination-defective meiotic mutants on fourth-chromosome crossing over in Drosophila melanogaster. Genetics 90: 699-712.

39.. Chen JM, Cooper DN, Chuzhanova N, Férec C, Patrinos GP (2009) Gene conversion in evolution and disease Encyclopedia of Life Sciences (ELS). Chichester: John Wiley \& Sons, Ltd.

40. Burger A, Lindsay H, Felker A, Hess C, Anders C, Chiavacci E, Zaugg J, Weber LM, Catena R, Jinek M, et al (2016) Maximizing mutagenesis with solubilized CRISPR-Cas9 ribonucleoprotein complexes. Dev Camb Engl 143: 2025-2037. doi:10.1242/dev.134809

41. Meccariello A, Monti SM, Romanelli A, Colonna R, Primo P, Inghilterra MG, Del Corsano G, Ramaglia A, lazzetti G, Chiarore A, et al (2017) Highly efficient DNA-free gene disruption in the agricultural pest Ceratitis capitata by CRISPR-Cas9 ribonucleoprotein complexes. Sci Rep 7: 10061. doi:10.1038/s41598-017-10347-5

42. Zhimulev IF, Belyaeva ES, Fomina OV, Protopopov MO, Bolshakov VN (1986) Cytogenetic and molecular aspects of position effect variegation in Drosophila melanogaster. Chromosoma 94: 492-504. doi:10.1007/ bf00292759

43. Elgin SCR, Reuter G (2013) Position-effect variegation, heterochromatin formation, and gene silencing in Drosophila. Cold Spring Harb Perspect Biol 5: a017780. doi:10.1101/cshperspect.a017780

44. Doheny JG, Mottus R, Grigliatti TA (2008) Telomeric position effect: A third silencing mechanism in eukaryotes. PLoS One 3: e3864. doi:10.1371/ journal.pone.0003864

45. Schoeftner S, Blasco MA (2009) A 'higher order' of telomere regulation: Telomere heterochromatin and telomeric RNAs. EMBO J 28: 2323-2336. doi:10.1038/emboj.2009.197

46. Wallrath LL, Elgin SC (1995) Position effect variegation in Drosophila is associated with an altered chromatin structure. Genes Dev 9: 1263-1277. doi:10.1101/gad.9.10.1263

47. Domínguez M, Brunner M, Hafen E, Basler K (1996) Sending and receiving the hedgehog signal: Control by the Drosophila gli protein cubitus interruptus. Science 272: 1621-1625. doi:10.1126/science.272.5268.1621 
48. Hartenstein V (1995) Atlas of Drosophila Development. Cold Spring Harbor, NY: Cold Spring Harbor Laboratory Press.

49. Port F, Chen HM, Lee T, Bullock SL (2014) Optimized CRISPR/Cas tools for efficient germline and somatic genome engineering in Drosophila. Proc Natl Acad Sci USA 111: E2967-E2976. doi:10.1073/pnas.1405500111

50. Port F, Bullock SL (2016) Augmenting CRISPR applications in Drosophila with tRNA-flanked sgRNAs. Nat Methods 13: 852-854. doi:10.1038/ nmeth.3972

51. Bischof J, Maeda RK, Hediger M, Karch F, Basler K (2007) An optimized transgenesis system for Drosophila using germ-line-specific phic31 integrases. Proc Natl Acad Sci USA 104: 3312-3317. doi:10.1073/ pnas.0611511104

52. Crucs S, Chatterjee S, Gavis ER (2000) Overlapping but distinct RNA elements control repression and activation of nanos translation. Mol Cell 5: 457-467. doi:10.1016/s1097-2765(00)80440-2

53. Wang C, Dickinson LK, Lehmann R (1994) Genetics of nanos localization in Drosophila. Dev Dyn 199: 103-115. doi:10.1002/aja.1001990204

54. Bhat KM (1999) The posterior determinant gene nanos is required for the maintenance of the adult germline stem cells during Drosophila oogenesis. Genetics 151: 1479-1492.

55. Liu X, Homma A, Sayadi J, Yang S, Ohashi J, Takumi T (2016) Sequence features associated with the cleavage efficiency of CRISPR/Cas9 system. Sci Rep 6: 19675. doi:10.1038/srep19675

56. Dang DT, Perrimon N (1992) Use of a yeast site-specific recombinase to generate embryonic mosaics in Drosophila. Dev Genet 13: 367-375. doi:10.1002/dvg.1020130507

57. Struhl G, Basler K (1993) Organizing activity of wingless protein in Drosophila. Cell 72: 527-540. doi:10.1016/0092-8674(93)90072-x

58. Tschopp P, Duboule D (2014) The genetics of murine Hox loci: TAMERE, STRING, and PANTHERE to engineer chromosome variants. Methods Mol Biol 1196: 89-102. doi:10.1007/978-1-4939-1242-1_6
59. Cunningham TJ, Lancman JJ, Berenguer M, Dong PDS, Duester G (2018) Genomic knockout of two presumed forelimb Tbx5 enhancers reveals they are nonessential for limb development. Cell Rep 23: 3146-3151. doi:10.1016/j.celrep.2018.05.052

60. Ma H, Marti-Gutierrez N, Park SW, Wu J, Lee Y, Suzuki K, Koski A, Ji D, Hayama T, Ahmed R, et al (2017) Correction of a pathogenic gene mutation in human embryos. Nature 548: 413-419. doi:10.1038/ nature23305

61. Grell EH (1961) The tetrasomic for chromosome 4 in Drosophila melanogaster. Genetics 46: 1177-1183.

62. Kosicki M, Tomberg K, Bradley A (2018) Repair of double-strand breaks induced by CRISPR-Cas9 leads to large deletions and complex rearrangements. Nat Biotechnol 36: 765-771. doi:10.1038/nbt.4192

63. Yagi R, Mayer F, Basler K (2010) Refined LexA transactivators and their use in combination with the Drosophila Gal4 system. Proc Natl Acad Sci USA 107: 16166-16171. doi:10.1073/pnas.1005957107

64. Markstein M, Pitsouli C, Villalta C, Celniker SE, Perrimon N (2008) Exploiting position effects and the gypsy retrovirus insulator to engineer precisely expressed transgenes. Nat Genet 40: 476-483. doi:10.1038/ng.101

65. Holtzman S, Kaufman T (2013) Large-scale imaging of Drosophila melanogaster mutations. FlyBase Reference Report (Last updated 2013). Accessed on 2013.

66. Bridges CB (1916) Non-disjunction as proof of the chromosome theory of heredity (concluded). Genetics 1: 107-163.

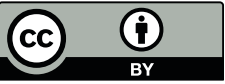

License: This article is available under a Creative Commons License (Attribution 4.0 International, as described at https://creativecommons.org/ licenses/by/4.0/). 